\section{All-optical wavelength con- version for polarization shift keying signal based on four-wave mixing in a semiconductor optical amplifier}

\author{
Liuyan Han, He Wen, Hanyi Zhang, and Yili Guo \\ Tsinghua University, Department of Electronic Engineering, \\ Beijing 100084, China \\ E-mail: hanly03@mails.tsinghua.edu.cn
}

\begin{abstract}
We propose an all-optical wavelength conversion method that can preserve the polarization information of an original signal based on four-wave mixing in a semiconductor optical amplifier. Using this method, we experimentally demonstrate wavelength conversion for a $10-\mathrm{Gb} / \mathrm{s}$ polarization shift keying signal with $1.6-\mathrm{dB}$ power penalty at a $10^{-9}$ bit error rate. To our knowledge, it is the first experiment reported on all-optical wavelength conversion for a polarization shift keying format. The converted polarization shift keying signal is successfully transmitted over a $75-\mathrm{km}$ standard single mode fiber with 1.8-dB transmission penalty. () 2007 Society of Photo-Optical Instrumentation Engineers. [DOI: 10.1117/1.2775936]
\end{abstract}

Subject terms: optical communication; all-optical wavelength conversion; polarization shift keying; four-wave mixing; semiconductor optical amplifier.

Paper 070342LR received Apr. 22, 2007; revised manuscript received Jun. 10, 2007; accepted for publication Jun. 15, 2007; published online Sep. 10, 2007.

\section{Introduction}

Polarization modulation techniques have been proposed and demonstrated in digital fiber communication. Polarization shift keying (PolSK), which uses the state of polarization of a fully polarized lightwave carrier as the information-bearing parameter, has been studied extensively and proven to have some advantages. ${ }^{1-4}$ Binary PolSK sensitivity has 3.4-dB improvement compared with intensity-modulation, direct-detection (IM/DD) systems. $^{1}$ PolSK features high insensitivity to laser phase noise, ${ }^{2}$ and it is more resistant to nonlinear fiber effects, because of the constant power. ${ }^{3}$ For all of these reasons, PolSK is considered a possible alternative to traditional modulation formats. Furthermore, polarization modulation techniques have also been utilized to enhance performance of existing modulation methods. For example, an alternate-polarized return-to-zero (APRZ) signal with orthogonal polarization between adjacent bits shows significant improvement in long transmission quality compared with nonreturn-to-zero (NRZ) and RZ formats.,

Wavelength conversion techniques are very important for the implementation of future all-optical networks. The study of wavelength conversion for polarization modulated signals is expected, so that new modulation formats, such

0091-3286/2007/\$25.00 @ 2007 SPIE

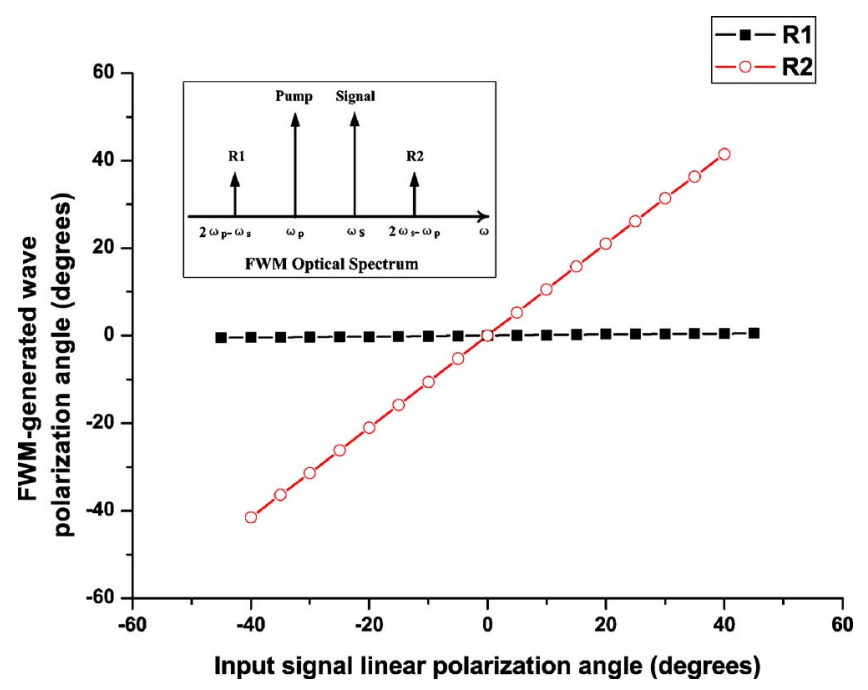

Fig. 1 Polarization angles of $\mathrm{R} 1$ and $\mathrm{R} 2$ versus the input signal polarization angle. Inset: schematic diagram of optical spectrum generated by FWM in a SOA.

as PolSK and APRZ, can be applied in future high-capacity all-optical networks. But it is difficult to preserve the original polarization information of an input signal by using the traditional wavelength conversion schemes, such as those based on cross-gain modulation (XGM) and cross-phase modulation (XPM). Until now, no study of all-optical wavelength conversion for polarization modulated signals has been carried out, to our knowledge.

In this letter, a simple and effective method of wavelength conversion for polarization modulated signals is proposed, which makes use of polarization property of fourwave mixing (FWM) in a semiconductor optical amplifier (SOA). Wavelength conversion for PolSK format is experimentally investigated. A $10-\mathrm{Gb} / \mathrm{s}$ PolSK signal is successfully converted with 1.6-dB penalty. The converted PolSK signal is transmitted over a $75-\mathrm{km}$ standard single mode fiber (SMF) and shows about 1.8-dB transmission penalty.

\section{Principle}

The four-wave mixing process in SOA is strongly related to the polarization states of the optical waves involved. The polarization state of the FWM-generated signal depends on those of the input waves. We make use of this principle to realize wavelength conversion for polarization modulated signals.

We assume that a pump light at the frequency $\omega_{p}$ is injected into a SOA together with a signal light $\left(\omega_{s}\right)$. A typical FWM optical spectrum is schematically presented in the inset of Fig. 1. R1 and R2 are products of the FWM process, at frequencies $\omega_{R 1}=2 \omega_{p}-\omega_{s}$ and $\omega_{R 2}=2 \omega_{s}-\omega_{p}$. Generally, R1 is utilized as a converted signal in traditional wavelength conversion schemes. However, in our scheme, $\mathrm{R} 2$ is utilized as the converted signal instead of R1, because R2 will preserve the polarization information of the input signal, but R1 will not.

The FWM process in an SOA can be explained by beating between the same polarization components of input 


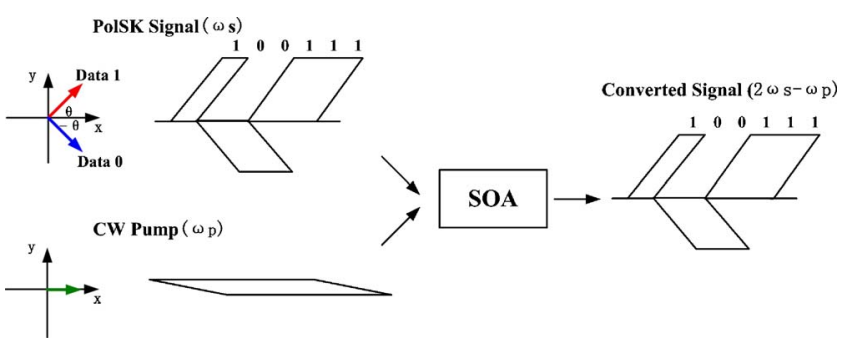

Fig. 2 Schematic diagram of a wavelength converter for the PoISK signal.

pump and signal. Using a simple lumped model for FWM in $\mathrm{SOA}^{7}$ the fields of FWM-generated waves can be written as

$\hat{E}_{\mathrm{R} 1}=\left(\hat{E}_{p} \cdot \hat{E}_{s}^{*}\right) r\left(\omega_{p}-\omega_{s}\right) \hat{E}_{p}$,

$\hat{E}_{\mathrm{R} 2}=\left(\hat{E}_{s} \cdot \hat{E}_{p}^{*}\right) r\left(\omega_{p}-\omega_{s}\right) \hat{E}_{s}$,

where $\hat{E}_{p}, \hat{E}_{s}, \hat{E}_{R 1}$, and $\hat{E}_{R 2}$ are vectors representing the fields of the pump, the signal, and R1 and R2, respectively. $r$ is the relative conversion efficiency function.

According to Eqs. (1) and (2), FWM-generated R1 and $\mathrm{R} 2$ possess the same polarization state as the pump and the signal, respectively. So the polarization information in the signal can be preserved by $\mathrm{R} 2$.

Next, simulation is performed with a commercialized Virtual Prototyping Inc. (VPI) tool. We fix the polarization angle of the input pump at $0 \mathrm{deg}$ and change the input signal polarization. Figure 1 plots the measured polarization angles at frequencies of R1 and R2 after SOA. The simulation results show that the polarization state of $\mathrm{R} 1$ is hardly varied, but the polarization state of R2 depends on the input signal polarization state.

Figure 2 is the schematic diagram of our proposed wavelength converter for a PolSK signal. A PolSK signal and a continuous-wave pump beam are injected into the SOA. The FWM signal at $\omega_{R 2}=2 \omega_{s}-\omega_{p}$ will be modulated in the polarization state as the input signal. Considering that FWM efficiency is dependent on the polarization angle between input optical waves, to obtain constant power for a converted PolSK signal, the polarization angle between pump light and the logic " 0 " state of the input PolSK signal should be equal to that between the pump and "1" state of the signal.

\section{Experimental Setup and Results}

The experimental setup is shown in Fig. 3. The PolSK signal is generated based on differential phase-to-polarization conversion in a differential group delay (DGD) element. ${ }^{8} \mathrm{~A}$ $\mathrm{LiNbO}_{3}$ phase modulator is used to encoding a continuouswave light at $1546.5 \mathrm{~nm}$ with $10-\mathrm{Gb} / \mathrm{s}$ differential phase shift keying (DPSK) format. Then a polarization controller (PC) is used to adjust the input signal polarization at $45 \mathrm{deg}$ to DGD (from General Photonics Corporation, Los Angeles, California) principle axes. After a 90-ps DGD, the DPSK signal is converted into a PolSK signal. Another laser source provides pump light at $1548.5 \mathrm{~nm}$. Pump and PolSK signal lights are combined after amplification and

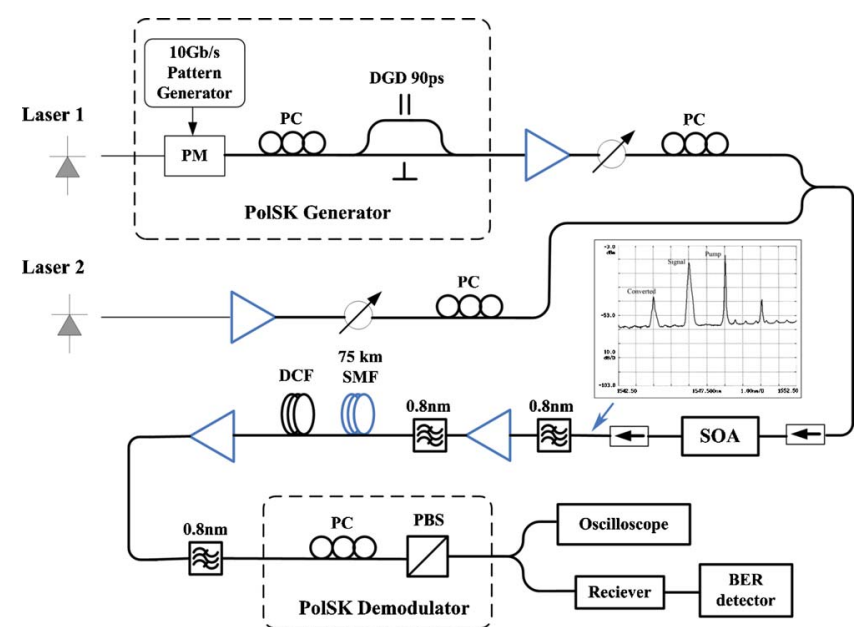

Fig. 3 The experimental setup. Inset: output optical spectrum from the SOA.

launched into SOA. The input signal and pump power are 1.6 and $-0.2 \mathrm{dBm}$, respectively. Two PCs are used to independently adjust the input pump and signal polarizations so that a constant power of converted signal can be achieved. The used SOA is manufactured by the Centre for Integrated Photonics Ltd. (Ipswitch, UK) (CIP) with a small signal gain of $30 \mathrm{~dB}$. The saturation output power is $9.6 \mathrm{dBm}$. The polarization-dependent saturated gain (PDG) of the SOA is less than $0.5 \mathrm{~dB}$. The optical spectrum after SOA is shown in the inset of Fig. 3. The converted signal at $1544.5 \mathrm{~nm}$ is selected by a $0.8-\mathrm{nm}$ optical filter and amplified. Then the converted signal is launched into a transmission link made of $75-\mathrm{km}$ SMF and a section of dispersion compensation fiber (DCF). At the receiver end, the PolSK signal is amplified and demodulated to an ASK signal by a $\mathrm{PC}$ and a passive polarization beamsplitter (PBS).

The experiment results are shown in Fig. 4, including the waveforms and eye diagrams of the input PolSK signal [Fig. 4(a)] and converted signal before transmission [Fig. 4(b)]. The upper line is the PolSK waveform before demodulation. The middle section is the waveform after demodulation. The lower section is the eye diagram after demodulation. Due to the constant power nature of the input PolSK signal, pattern-dependent degradation in SOA is reduced. The converted PolSK signal mainly suffers from signal-to-noise ratio (OSNR) degradation caused by low FWM conversion efficiency and ASE of SOA.

In bit-error-rate (BER) measurements, we make use of the PRBS property to avoid any electronic differential encoder. The obtained results are shown in Fig. 5, including BER curves of the converted PolSK signal before and after 75-km transmission, together with back-to-back measurements. It can be seen in Fig. 5 that the wavelength conversion leads to a penalty of about $1.6 \mathrm{~dB}$ at a BER of $10^{-9}$. A demodulated eye diagram for the converted signal after transmission is displayed in the inset of Fig. 5. The transmission penalty is around $1.8 \mathrm{~dB}$. Since our experiment setup is composed of separate devices and the fiber in the link produces an unknown fluctuation of the polarization state, the converted PolSK signal polarization state will drift. This will degrade the BERs a little or more. If a 


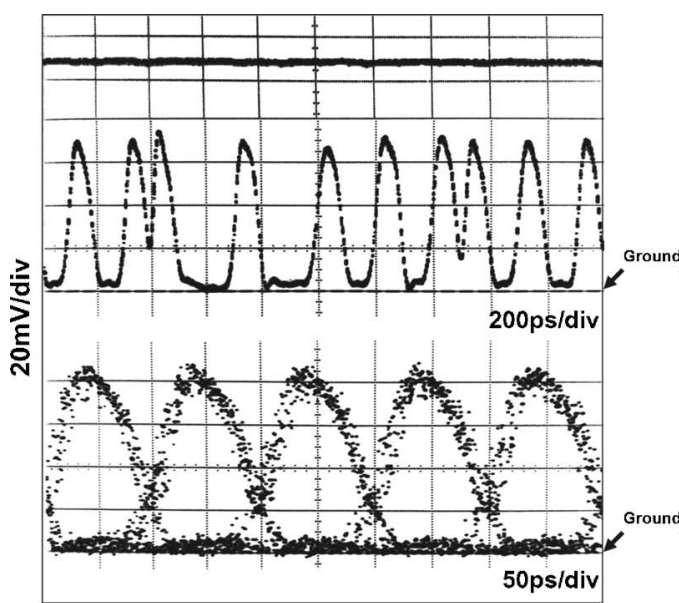

(a)

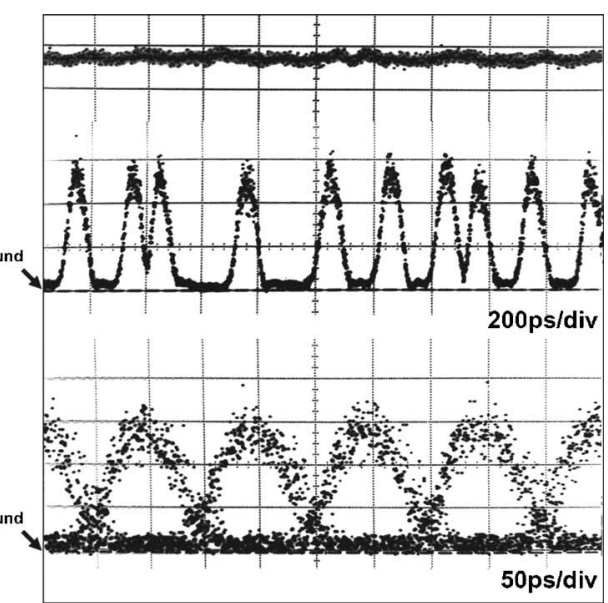

(b)

Fig. 4 Waveforms and eye diagrams of (a) input PolSK signal and (b) converted signal. Top: PolSK waveform before demodulation. Middle: waveform after demodulation. Bottom: eye diagram after demodulation.

dynamic polarization control is used to compensate the polarization fluctuations at the demodulating part, BER performance may be improved.

In our scheme, to obtain a converted signal with constant power, the input wave polarization states into SOA need to be adjusted as previously mentioned. Considering that in practical applications the polarization state of an input signal may suffer a random and dynamic change after fiber transmission, to avoid dynamically adjusting the input polarization states into SOA, we can adopt some techniques to realize polarization-insensitive FWM conversion efficiency, such as dividing the input pump into two equal power components that are polarization orthogonal and noncoherent with each other by using the scheme in Ref. 9 .

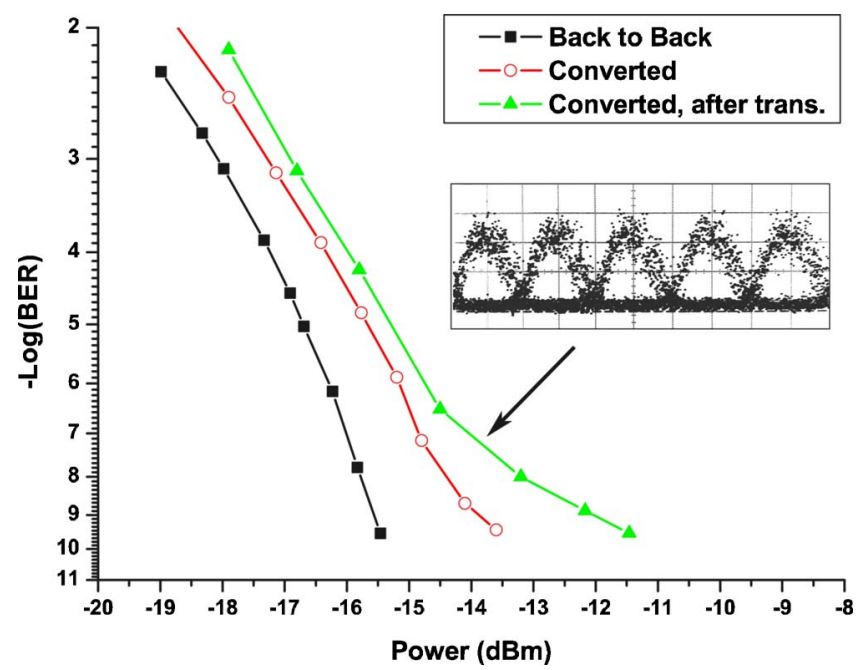

Fig. 5 BER measurements for PoISK wavelength conversion at $10 \mathrm{~Gb} / \mathrm{s}$. Inset: demodulated eye diagram for the converted signal after $75-\mathrm{km}$ transmission.

\section{Conclusion}

We demonstrate wavelength conversion for PolSK format based on four-wave mixing in SOA. Error-free wavelength conversion at $10 \mathrm{~Gb} / \mathrm{s}$ is obtained. The converted signal after $75-\mathrm{km}$ fiber transmission shows an overall power penalty of $3.4 \mathrm{~dB}$ relative to the back-to-back case. The proposed method has a potential for higher speed wavelength conversion, and it may be performed for other optical modulation formats that utilize polarization modulation techniques.

\section{Acknowledgment}

This work was supported by the National Natural Science Foundation of China (NSFC number 60520130298).

\section{References}

1. S. Benedetto, R. Gaudino, and P. Poggiolini, "Direct detection of optical digital transmission based on polarization shift keying modulation," IEEE J. Sel. Areas Commun. 13(3), 531-542 (1995).

2. S. Benedetto and P. Poggiolini, "Theory of polarization shift keying modulation," IEEE Trans. Commun. 40(4), 708-721 (1992).

3 . E. Hu, Y. Hsueh, K. Shimizu, K. Wong, N. Kikuchi, M. Marhic, and L. Kazovsky, "4-level direct-detection polarisation shift-keying (DDPolSK) system with phase modulators," OFC 2003, Vol. 2, pp. 647 649 (2003).

4. C. W. Chow, C. S. Wong, and H. K. Tsang "Optical packet labeling based on simultaneous polarization shift keying and amplitude shift keying," Opt. Lett. 29(16), 1861-1863 (2004).

5. A. Hodzic, B. Konrad, and K. Petermann, "Improvement of system performance in $\mathrm{N} \times 40-\mathrm{Gb} / \mathrm{s}$ WDM transmission using alternate polarizations," IEEE Photonics Technol. Lett. 15(1), 153-155 (2003).

6. C. Xie, I. Kang, A. H. Gnauck, L. Moller, L. F. Mollenauer, and A. R. Grant, "Suppression of intra-channel nonlinear penalties in highspeed transmissions with alternate polarization formats," OFC 2004 Postconf. Digest, pp. 310-312 (2004).

7. J. P. R. Lacey, M. A. Summerfield, and S. J. Madden, "Tunability of polarization-insensitive wavelength converters based on four-wave mixing in semiconductor optical amplifiers," J. Lightwave Technol. 16(12), 2419-2427 (1998).

8. E. Ciaramella, G. Contestabile, and A. D'Errico, "A novel scheme to detect optical DPSK signals," IEEE Photonics Technol. Lett. 16(9), 2138-2140 (2004).

9. T. Yang, C. Shu, and C. Lin, "Depolarization technique for wavelength conversion using four-wave mixing in a dispersion-flattened photonic crystal fiber," Opt. Express 13(14), 5409-5415 (2005). 